\title{
A NOVEL TECHNIQUE TO SALVAGE FAILING TYMPANOPLASTY- A PROSPECTIVE INTERVENTIONAL TRIAL
}

\author{
Subhradev Biswas ${ }^{1}$, Ankit Choudhary2, Sayan Hazra ${ }^{3}$, Kaustuv Das Biswas ${ }^{4}$, Bijan Basak ${ }^{5}$
}

${ }_{1}^{1}$ Assistant Professor, Department of ENT, Head and Neck Surgery, IPGMER \& SSKM Hospital, Kolkata, West Bengal, India. ${ }^{2}$ Senior Resident, Department of ENT, Head and Neck Surgery, IPGMER \& SSKM Hospital, Kolkata, West Bengal, India. 3 Senior Resident, Department of ENT, Head and Neck Surgery, IPGMER \& SSKM Hospital, Kolkata, West Bengal, India. ${ }^{4}$ RMO CUm Clinical Tutor, Department of ENT, Head and Neck Surgery, IPGMER \& SSKM Hospital, Kolkata, West Bengal, India. ${ }_{5}^{5}$ Associate Professor, Department of ENT, Head and Neck Surgery, IPGMER \& SSKM Hospital, Kolkata, West Bengal, India.

\section{ABSTRACT}

\section{BACKGROUND}

This article describes a procedure to salvage graft failures in cases of Tympanoplasty done using Temporalis Fascia by early intervention, Post operatively.

\begin{abstract}
METHODS
It was an Observational study using 30 patients with residual perforations on $3^{\text {rd }} 6^{\text {th }}$ post-operative week out of 131 patients who underwent Type 1 Tympanoplasty with Cortical Mastoidectomy using Temporalis fascia as graft material. Study was conducted over a period of 18 months. Patients having residual perforation underwent the procedure after proper informed consent. 4 Quadrant blocks were given in External Auditory canal. The Epithelialized margin was freshened using a Micropick and medialised graft was repositioned after placing of a piece of Gelfoam in middle ear. The perforation was reinforced with a Gelfoam laterally in External Auditory Canal. Patient was then followed up with a course of Antibiotic and antihistaminic.
\end{abstract}

\section{RESULTS}

Out of 131 patients of cortical mastoidectomy with tympanoplasty 30 had residual perforation on follow up i.e., $77 \%$ success rate while $23 \%$ failure rate in our hand. These $23 \%$ patients, who were found to be failing, underwent this intervention. 24 out of these 30 patients $(80 \%)$ i.e., $18 \%$ of overall cases showed healed drum after intervention on further follow up. Thus, a good number of failing Tympanoplasty could be salvaged and overall success achieved is almost $95 \%$ as compared to $77 \%$ previously.

\section{CONCLUSIONS}

With this procedure $80 \%$ of the failing Tympanoplasties could be salvaged increasing success rate from $77 \%$ to $95 \%$ thus increasing reliability of Temporalis Fascia Graft which was being replaced increasingly by cartilage graft for risk of Failures by Otologists these days.

HOW TO CITE THIS ARTICLE: Biswas S, Choudhary A, Hazra S, et al. A novel technique to salvage failing tympanoplasty- a prospective interventional trial. J. Evolution Med. Dent. Sci. 2019;8(21):1700-1703, DOI: 10.14260/jemds/2019/374

\section{BACKGROUND \\ Chronic suppurative otitis media (CSOM) constitutes a major} public health problem in children and adults in the developing world.1,2 It is an infection characterized by recurrent middle-ear discharge through a persistent tympanic membrane perforation. The disease is the most common childhood infectious disease worldwide, starting early in life. ${ }^{1}$ However, in the developing world, risk factors such as malnutrition, over-crowding, substandard hygiene, frequent upper respiratory tract infections, and underresourced healthcare compound the problems and make the disease prevalent among children and adults. ${ }^{3}$

Tympanoplasty is the procedure to eradicate disease in the middle ear along with reconstruction of Tympanic membrane and/ or Ossicular chain. ${ }^{4}$

'Financial or Other Competing Interest': None.

Submission 30-03-2019, Peer Review 14-05-2019,

Acceptance 20-05-2019, Published 27-05-2019.

Corresponding Author:

Ankit Choudhary,

\#302, APC Road, Ideal Heights,

Block-C, Flat-8A,

Kolkata-700009,

West Bengal, India.

E-mail: dr.ankitchoudhary@gmail.com

DOI: $10.14260 / j e m d s / 2019 / 374$

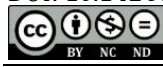

It is done in cases of Chronic Otitis media with perforation and is one of the commonest ENT procedures done. Surgical repair (Tympanoplasty) of the perforated tympanic membrane is indicated to restore hearing ability as well as to prevent recurrent otorrhea.

Since the introduction of tympanoplasty by Wullstein in 1952 and Zoellner in 1955, different types of graft materials have been used to reconstruct the Tympanic Membrane. These include temporalis fascia, skin, periostea, perichondrium, dura mater, cartilage, vein, and fat. Temporalis fascia remains the most commonly used material for tympanic membrane reconstruction, with a success rate of $93 \%$ to $97 \%$ in primary tympanoplasties. However, the situation gets more complex, and failure rates are considered higher in cases of Eustachian tube dysfunction, retraction pocket, adhesive otitis media, and subtotal or total perforation. Therefore, graft materials that are more rigid than fascia (i.e., cartilage) and more resistant to infection, resorption, and retraction have been proposed as more appropriate for tympanic membrane reconstruction. ${ }^{2}$ Various other graft materials have been employed in due course of time but advantages of using Temporalis fascia over others is manifold including local availability, good audiological outcome and Low BMR increasing its survival. Even with so many advantages the procedure is not full proof and success rate varies from $70-95 \%$ which doesn't depend only on surgical expertise. ${ }^{5}$ 

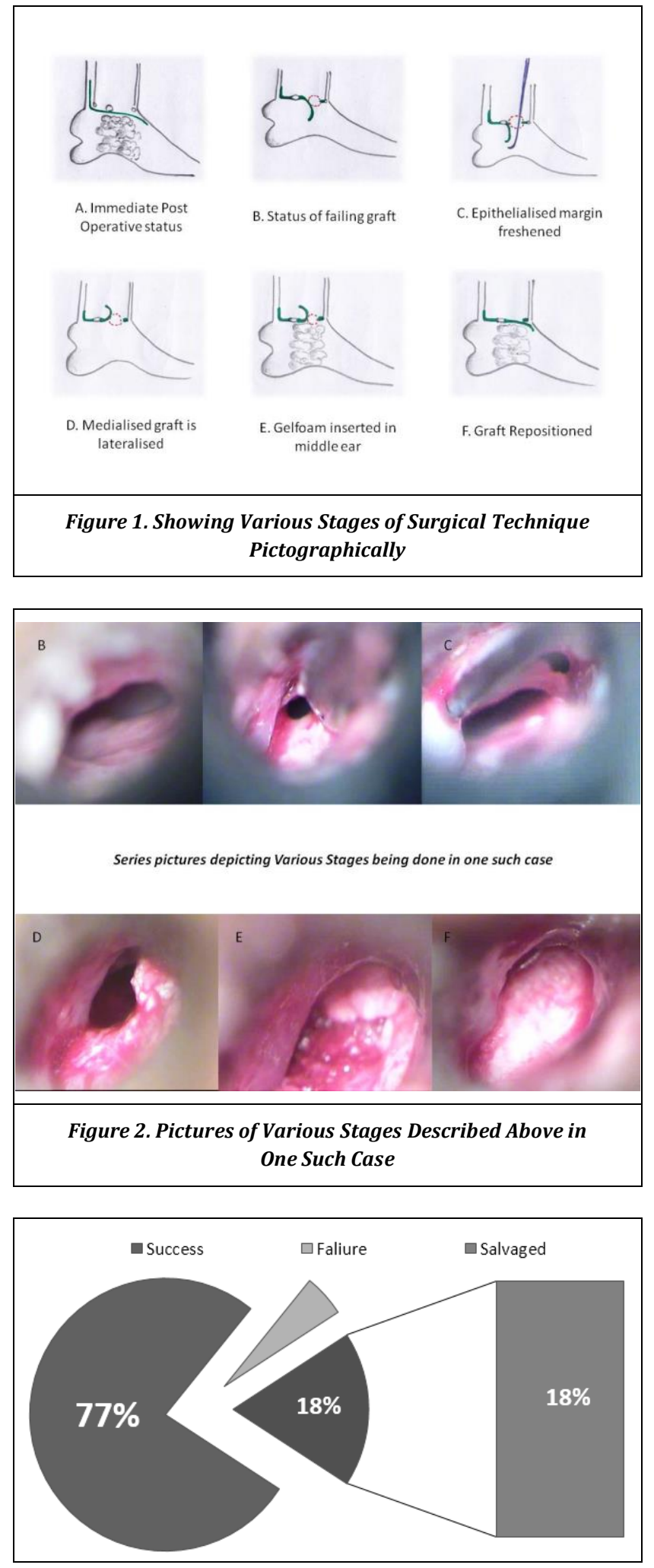

As per available literature forcing Otologists to switch to other graft materials like Cartilage with better success rate but poor audiological outcome. $6,7,8$

Various techniques have been used in failed cases of Tympanoplasty including office procedure using Trichloroacetic acid, ${ }^{9}$ fat patching, ${ }^{10}$ revision surgery using perichondrium or various other graft material with varying results. None of the technique used so far is a full proof method. Also, the mere thought of undergoing a second surgery imparts a big psychological pressure on the patient. Thus, in this Study we would like to salvage the failing
Tympanoplasty done using Temporalis fascia as graft material with the same failing graft itself thus increasing its overall success rate along with the good audiological outcome, i.e., increasing reliability of Temporalis Fascia Graft in Tympanoplasty.

\section{Aims and Objectives}

1. To salvage graft failures in cases of Tympanoplasty done using Temporalis Fascia.

2. To increase the reliability of Temporalis fascia graft.

3. Increase the overall success rate.

\section{METHODS}

It was an Observational study done in a Tertiary care centre. It was conducted on 30 patients between the age group of 16 yrs to 52 yrs of both the sexes. The study was done over a period of 18 months.

131 patients underwent Type 1 Tympanoplasty with Cortical Mastoidectomy using Temporalis fascia as graft material. In all the patients Moderate to Large central perforation was present involving the Pars Tensa. Type 1 tympanoplasty was done by Wilde's Post Aural approach using Temporalis fascia as graft material placed by Underlay technique after proper clearance of disease from middle ear with anterior support of Graft by Gelfoam. Wide cortical mastoidectomy was done and Ventilation was established in all the cases by clearing granulations/disease in attic/ aditus/ antrum if any. External Auditory Canal of the 131 patients were followed for 6 months post-surgery.

30 Cases were found to have residual perforations on post-operative visit. The below mentioned procedure was done as soon as a residual defect was detected in all the 30 cases on post-operative visits (Earliest being done at $3^{\text {rd }}$ week and Latest at $6^{\text {th }}$ Post-operative week). No residual perforation was detected post $6^{\text {th }}$ weeks in our study (Up to 6 months). Late or delayed graft failure could not be studied. Proper Informed consent for the procedure was taken from all the patients. Standard operating protocol for Tympanoplasty is described below along with detailed surgical technique of the procedure done to salvage graft failure.

\section{Tympanoplasty Procedure}

The edges of the perforation are freshened. The tympanomeatal flap is elevated from 6 0'clock to 12 0'clock position. Ossicular intactness and mobility are confirmed. The temporalis fascia graft is harvested through an extended postaural incision or through a separate $2 \mathrm{~cm}$ incision in the temporal region of scalp after infiltrating with $2 \%$ lignocaine and 1:100, 000 adrenalin. The graft is then dried over a bowl of hot water. ${ }^{1}$ After putting antibiotic steroid-soaked gel foam in the middle ear, the graft is placed medial to the handle of malleus and carefully placed below the perforation. The tympanomeatal flap is repositioned. The final graft position is checked and readjusted if required. The external auditory canal is filled with antibiotic steroid-soaked gel foam to stabilize the graft. A small ear pack soaked with antibiotic is kept in the ear canal. Incision is closed and mastoid dressing given. IV fluids, IV antibiotics, and IV analgesics were given. Post-operative complications such as facial nerve weakness/palsy, soakage of mastoid dressing, vertigo, and nystagmus were observed. Antibiotic, analgesic, 
decongestants, and antihistamines will be given for 1 week. Steroid nasal spray and mast cell stabilizers were continued in those patients with nasal allergy. Patients were advised not to cough, strain, or sneeze and keep ears dry. All patients were instructed to avoid air travel and swimming for 1 month. Postaural suture removal was done on the 7 th postoperative day. All patients were called for regular follow-up. The gel foam in the external auditory canal was not disturbed for 3 weeks. Antibiotic ear drops were started to facilitate dissolution of gel foam and to promote healing. All patients were followed up at regular intervals at the end of the after surgical procedure. Of 1 month. Any residual gel foam is cleared by gentle suctioning. The underlay technique is widely used and is relatively simple to perform, as the graft is placed entirely medial to the remaining drum and malleus ${ }^{3}$. In underlay type I tympanoplasty the graft is placed below all the three layers. Thus, there are chances of graft medialization as well as danger of residual perforation Underlay technique is considered easier and faster technique with a high graft uptake rate.

\section{Surgical Technique}

Examination under Microscope was done in all the follow up patients of Tympanoplasty on or after third postoperative week. During Examination under Microscope, Suction Clearance was done to clear all the residual Gelfoam. Proper evaluation of Graft, Neotympanum and Residual perforation was done. Residual perforation was found to be involving anterior or anteroinferior quadrant of Neotympanum in all 30 out of 131 cases. None of the case was found having Posterior residual perforation in our study. Careful evaluation revealed Tympanic Membrane margin at the site of residual perforation to be re-epithelialised and graft margin to be anteriorly medialized (B). 4 Quadrant local anesthetic block was given in External Auditory Canal of these patients. The Epithelialised margin of residual perforation is freshened using a Micropick (C) and then the Medialised margin of the graft lateralised (D). A piece of Gelfoam is now placed in middle ear (E) and lateralised graft was then supported over it and repositioned (F). The perforation is then reinforced with Gelfoam laterally in External Auditory Canal. Patient was then followed up with a course of Antibiotic for a week and antihistaminic drugs for a period of one month.

\section{RESULTS}

Out of 131 patients of cortical mastoidectomy with tympanoplasty 30 had residual perforation on follow up i.e., $77 \%$ success rate while $23 \%$ failure rate in our hand. These $23 \%$ patients, who were found to be failing, underwent this intervention. 24 out of these 30 patients $(80 \%)$ i.e., $18 \%$ of overall cases showed healed drum after intervention on further follow up. Thus, a good number of failing Tympanoplasty could be salvaged and overall success achieved is almost $95 \%$ as compared to $77 \%$ previously.

In 2 of the failed cases graft was found to be short of defect but repositioning maneuver was tried and a great reduction in size of defect was seen.

\section{DISCUSSION}

Chronic otitis media (COM) is a result of the previous episode of acute otitis media, otitis media with effusion, or trauma to the tympanic membrane (TM). This causes a permanent defect of the pars tensa which leads to recurrent infection and ear discharge. Eventually, these patients may develop hearing loss and further complications. These patients can be managed surgically by doing a tympanoplasty. ${ }^{1}$

Tympanoplasty is classified by various authors. Wullstein and Zoellner popularised myringoplasty technique in 1951 for tympanic membrane perforations. Now with much improved optics and microsurgery techniques, it is still commonly practiced in modern days with most commonly used classification being of Wullstein only.

\section{Types of Tympanoplasty (Wullstein Classification ${ }^{11)-}$}

Type I: Middle ear practically restored to normal; tympanic membrane and middle ear intact.

Type II: Middle ear of approximately normal size is tried to preserve in spite of slight defects of the ossicles.

Type III: Large defects of the malleus and incus warrant the removal of the ossicular chain and of the epitympanum. The tympanic membrane must be directly connected with the head of the stapes.

Type IV: The stapedial footplate is movable, but the crurae are missing. The tympanoplasty reconstructs a middle ear comprising only of the tube and the hypotympanum with sound protection for the round window.

Type V: The stapedial footplate is fixed and a fenestra novovalis is necessary.

The main goals of tympanoplasty are removal of the active disease and restoration of damage due to sequelae. Various factors such as smoking, pathology in the contralateral ear, size of the tympanic membrane perforation, experience of the surgeon, and duration of the dry period have been reported to have an effect on the rates of success after tympanoplasty. Other factors supposed to be associated with the surgical outcome of tympanoplasty are age, gender, site of the perforation size, drainage status of the ear at the time of surgery and surgeon experience. However, the actual roles and effects of these factors remain controversial. Graft success is an important indicator after tympanoplasty and if the tympanic membrane heals during the postoperative period, improvement in hearing loss and prevention of recurrent middle ear infection may be expected.12

Type I tympanoplasty or myringoplasty repairs the perforation of tympanic membrane alone. The closure of perforation is achieved using different autologous graft materials ${ }^{1}$. Various graft materials have been used since then including skin, amniotic membrane, mucous membrane, dura mater, cornea, periosteum, perichondrium, vein, adipose tissue and temporalis fascia. Among the various options available otologists prefer to use temporalis fascia or perichondrium as it gives a good healing, sufficient quantity of graft, good tensile strength, and acoustic property similar to that of normal Tympanic Membrane. Unfortunately, fascia grafts are found to succumb to infections and significant pressure gradient during the post-operative period. This can be avoided in certain cases using a cartilage-perichondrial graft. ${ }^{1}$ While temporalis fascia has better functional outcome with respect to hearing, it is subject to poor dimensional stability. The poor dimensional stability of temporalis fascia 
grafts contributes to residual perforations following tympanoplasty, particularly in large tympanic membrane perforations. Palisade cartilage and cartilage island techniques have the disadvantage of interfering with the sound-conducting mechanism, and hence patients may benefit only minimally with regard hearing restoration. ${ }^{3}$

Even with the most experienced hand success rate of Tympanoplasty using Temporalis fascia graft is not $100 \%$. Often, we are forced to see a failing Tympanoplasty during follow up, about which we can't do anything. Only after failure is established, we employ various techniques like Trichloroacetic acid cautery, fat patching or even revision surgery. In this technique demonstrated above we can intervene before the procedure is a complete failure. Instead of watching the graft fail, we can save the valuable time as also the discomfort of failure and cost of revision surgery by using that failing graft itself as an OPD procedure.

Using this technique $80 \%$ of the failing Tympanoplasty could be salvaged. Results obtained in this technique was comparable to results of various available literature pertaining to other methods.8,9,10 No need of extra invasive procedure to harvest graft material is needed as we are utilizing the failing graft itself and performing the procedure early when the graft is still in failing phase are the added advantage over other techniques. Extra consultation and readmission are also avoided.

\section{CONCLUSIONS}

With this procedure, $80 \%$ of the failing Tympanoplasties could be salvaged increasing success rate from $77 \%$ to $95 \%$ thus increasing reliability of Temporalis Fascia Graft which was being replaced increasingly by cartilage graft for risk of Failures by Otologists these days.

\section{Limitations}

The procedure was limited to anterior/anteroinferior residual perforation only because in our series we failed to find residual perforation in any other quadrant. Secondly, availability of medialized graft in delayed diagnosis of perforation could not be established as all the cases were diagnosed in early $\left(3^{\text {rd }}-6^{\text {th }}\right)$ post-operative weeks only. In 2 cases, the graft was found to fall short of defect. It was repositioned leading to reduction in size of resultant perforation. Anterior elevation of annulus and anterior tucking beyond annulus was not done in primary surgery which itself could have reduced incidence of graft failure.

\section{REFERENCES}

[1] Aneesa AMF, Pillai A, Rajamma KB. Outcome of type 1 tympanoplasty with cartilage-perichondrium graft in comparison with temporalis. International Journal of Scientific Study 2019;6(10):62-7.

[2] Jalali MM, Motasaddi M, Kouhi A, et al. Comparison of cartilage with temporalis fascia tympanoplasty: a meta-analysis of comparative studies. Laryngoscope 2017;127(9):2139-48.

[3] Indorewala S, Adedeji TO, Indorewala $\mathrm{A}$, et al. Tympanoplasty outcomes: a review of 789 cases. Iran J Otorhinolaryngol 2015;27(79):101-8.

[4] Gleeson MJ, Clarke RC. Scott Brown's Otorhinolaryngology: head and neck surgery. Vol. 3. 7th edn. CRC Press 2008: p. 3421.

[5] Palva T, Ramsay H. Myringoplasty and tympanoplasty - results related to training and experience. Clinical Otolaryngology and Allied Sciences 1995;20(4):32935.

[6] Freitas MR, Oliveira TC. The role of different types of grafts in tympanoplasty. Brazilian Journal of Otorhinolaryngology 2014;80(4):275-6. https://dx.doi.org/10.1016/j.bjorl.2014.05.018

[7] Lee JC, Lee SR, Nam JK, et al. Comparison of different grafting techniques in type I tympanoplasty in cases of significant middle ear granulation. Otol Neurotol 2012;33(4):586-90.

[8] Zahnert T, Hottenbrink KB, Mürbe D, et al. Experimental investigations of the use of cartilage in tympanic membrane reconstruction. Am J Otol 2000;21(3):322-8.

[9] Derlacki EL. Residual perforations after tympanoplasty: office technique for closure. Otolaryngol Clin North Am 1982;15(4):861-7.

[10] Veetil SAS, Rajendran GC, Mukundan A, et al. Endoscopic fat grafting of small and residual tympanic membrane perforations - a clinical study. J Evolution Med Dent Sci 2017;6(50):3828-31.

[11] Merkus P, Kemp P, Ziylan F, et al. Classifications of mastoid and middle ear surgery: a scoping review. J Int Adv Otol 2018;14(2):227-32.

[12] YurttaS V, Ural A, Kutluhan A, et al. Factors that may affect graft success in tympanoplasty with mastoidectomy. ENT Updates 2015;5(1):9-12. 